\title{
AGGREGATION OF BIFUNCTIONAL
}

\section{EXTRACTANTS USED FOR URANIUM(VI)}

\section{SEPARATION}

Diego Moreno Martinez, Eléonor Acher, Marin Vatin, Sandrine Dourdain, Dominique

Guillaumont*, Philippe Guilbaud*.

CEA, DES, ISEC, DMRC, Univ Montpellier, Marcoule, France

*Philippe Guilbaud. Email: philippe.guilbaud@cea.fr, *Dominique Guillaumont. Email:

dominique.guillaumont@cea.fr

\section{Force fields}

For the $\mathrm{n}$-dodecane molecules and aliphatic chains of DEHCNPB, the bonds and angle parameters were taken from parm 99 force field. ${ }^{1}$ The dihedral parameters developed by Junmei et al. were used. ${ }^{2}$ The OPLS-AA parameters were used for non-bonded interactions. ${ }^{3}$

The missing parameters on the parm99 field for phosphonate were taken from gaff force field. ${ }^{4}$ The dihedral parameters of Kristin et al. were used. ${ }^{5}$

Calculated density and concentration of the simulation boxes

\begin{tabular}{|c|c|c|c|c|c|}
\hline \multicolumn{3}{|l|}{ Experiment } & \multicolumn{3}{|c|}{ MD simulations } \\
\hline $\begin{array}{l}\text { [DEHCNPB] in } \\
\text { mol.t. }^{-1}\end{array}$ & $\begin{array}{l}\text { Density in } \\
\text { g. } \mathrm{mL}^{-1}\end{array}$ & $\begin{array}{l}\text { Simulation } \\
\text { name }\end{array}$ & $\begin{array}{l}\text { Density in } \\
\text { g. } \mathrm{mL}^{-1}\end{array}$ & $\begin{array}{l}\text { [DEHCNPB] } \\
\text { in mol. } \mathrm{L}^{-1}\end{array}$ & $\begin{array}{l}\text { Box volume } \\
\text { in } \mathrm{nm}^{3}\end{array}$ \\
\hline 0.05 & 0.7551 & DEHCNPB_005 & 0.7412 & 0.049 & 1013.54 \\
\hline 0.10 & 0.7599 & DEHCNPB_01 & 0.7494 & 0.098 & 1017.10 \\
\hline 0.50 & 0.8042 & DEHCNPB_05 & 0.7973 & 0.494 & 1007.49 \\
\hline 0.80 & 0.8315 & DEHCNPB_08 & 0.8307 & 0.780 & 1021.15 \\
\hline- & - & DEHCNPB_01_U & 0.7610 & 0.101 & 985.28 \\
\hline
\end{tabular}

Table S.1. Volume of the boxes, as well as the calculated and experimental densities. DEHCNPB concentrations in the MD boxes are also depicted. 


\section{Calculated SAXS and SANS signals for DEHCNPB_01}
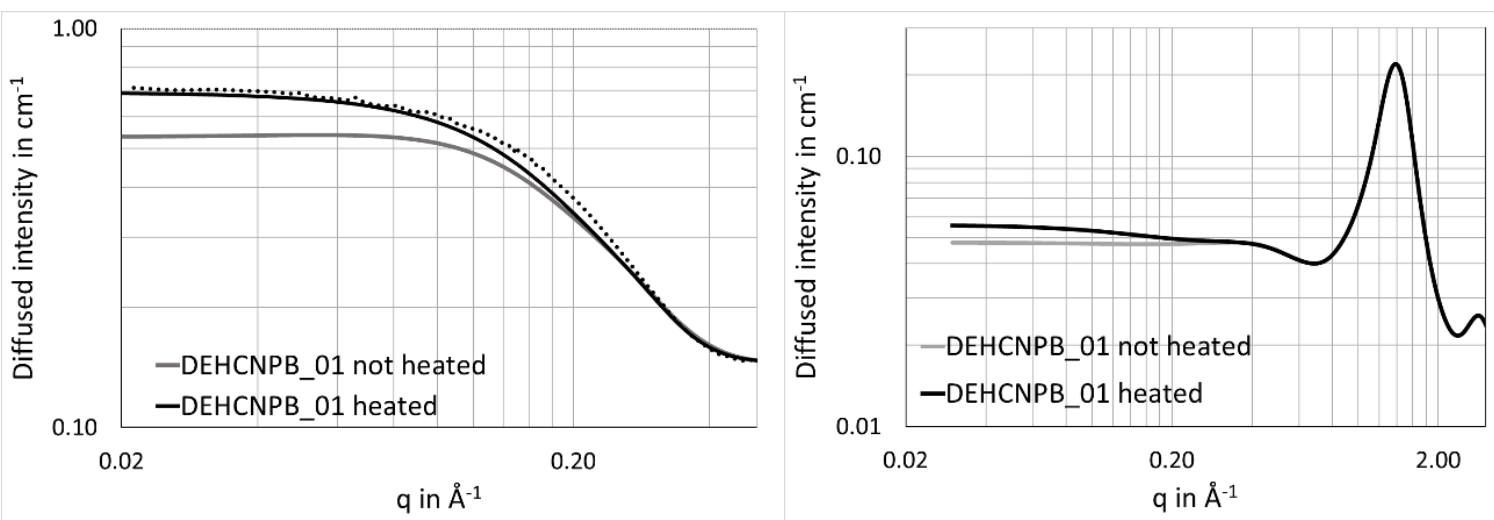

Figure S.1. Comparison of SAXS (right) and SANS (left) signals between the heated and not heated MD simulation for DEHCNPB_01. The dotted line on the SANS graph (left) corresponds to the experimental spectra.

\section{Aggregation number calculation}

The aggregation numbers were calculated as fallows:

$$
N_{a g}=\sum_{i} \frac{n_{i}}{n_{t o t}} i
$$

Were $\mathrm{n}_{\mathrm{i}}$ corresponds to the number of aggregates of size i ( 1 for monomers, 2 for dimers, etc.) and $n_{\text {tot }}$ corresponds to the total number of aggregates species.

\section{Definition of aggregate}

Computationally the aggregates are counted fallowing distances criteria. The choice of distances was based in characteristic peaks of interaction, found on Radial Distribution functions, for three pairs of atoms: the P-P, P-Ow and the Ow-Ow distances. Some RDF peaks are displaced in presence of uranyl. Consequently, criteria change whether the specie contains, or not, uranyl. Two molecules are considered in interactions if one of these conditions is true:

\begin{tabular}{ccc} 
Pair of atoms & $\begin{array}{c}\text { Distance criteria without } U \\
\text { in } \AA\end{array}$ & Distance criteria with $U$ in $\AA$ \\
\hline $\begin{array}{c}\text { P-P (for DEHCNPB-DEHCNPB } \\
\text { interactions) }\end{array}$ & $\leq 6.0$ & $\leq 8.0$ \\
\hline $\begin{array}{c}\text { P-Ow (for DEHCNPB-water } \\
\text { interactions) }\end{array}$ & $\leq 4.0$ & $\leq 5.0$ \\
\hline $\begin{array}{c}\text { Ow-Ow (for water-water } \\
\text { interactions) }\end{array}$ & $\leq 3.4$ & $\leq 3.4$
\end{tabular}


The criteria for DEHCNPB are available whatever the interaction is done by the phosphonate or amide groups. In the case of DEHCNPB_01_U simulation, the analysis of aggregates were realized twice, first for the species containing uranium, then for the species without uranium. This discrimination was done using another distance criterion: whether a uranium atom is found around $8.0 \AA$ of a P or Ow atom. A diagram of the logical procedure for counting aggregates is depicted in Figure S.2.

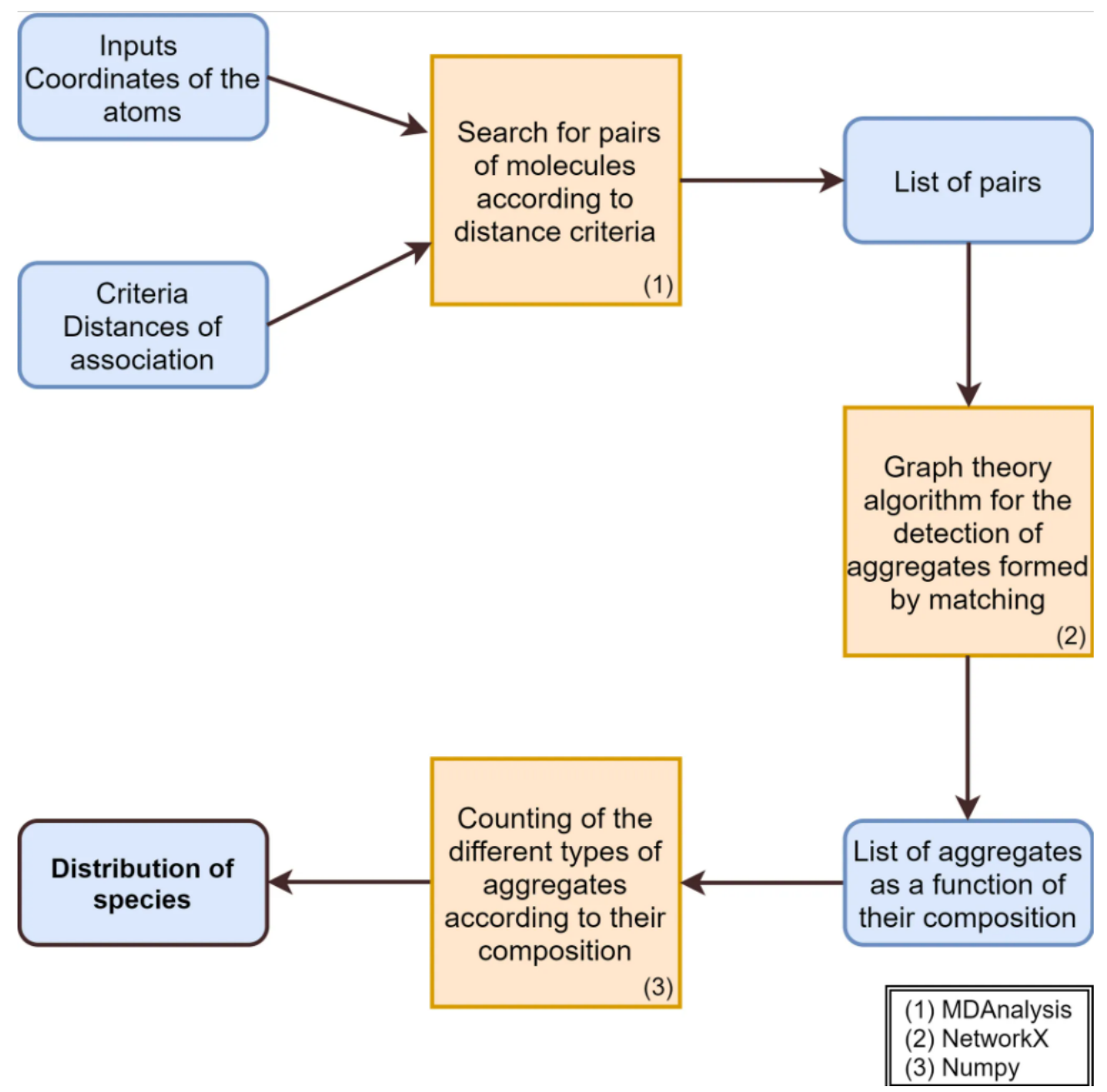

Figure S.2. Diagram of the Python procedure to count aggregates. The inputs and/or outputs are shown in blue, and the executed actions are in orange. The names of the python modules used in each action are specified. 


\section{Radial Distribution Functions (RDFs)}

\section{RDFs centred on uranium atoms}

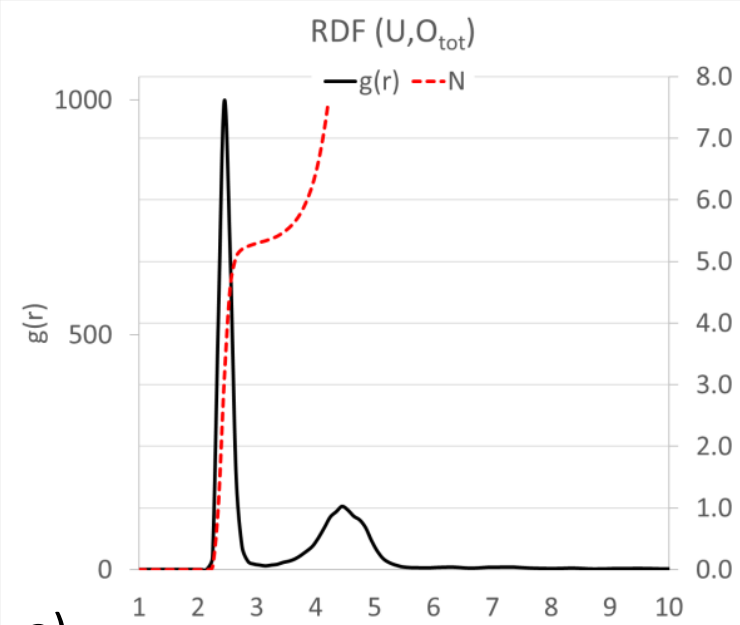

a)

$r(\AA ̊)$
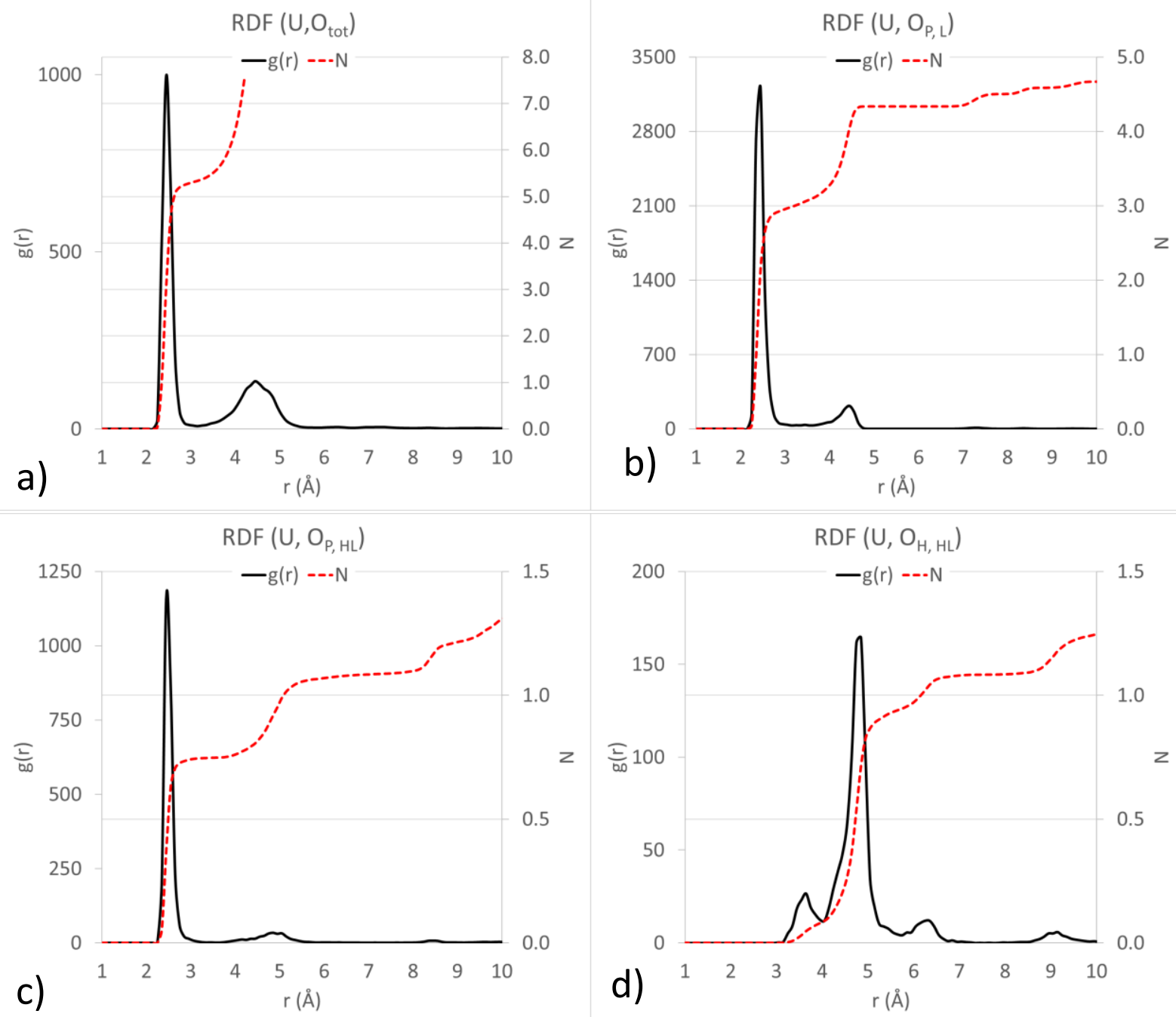


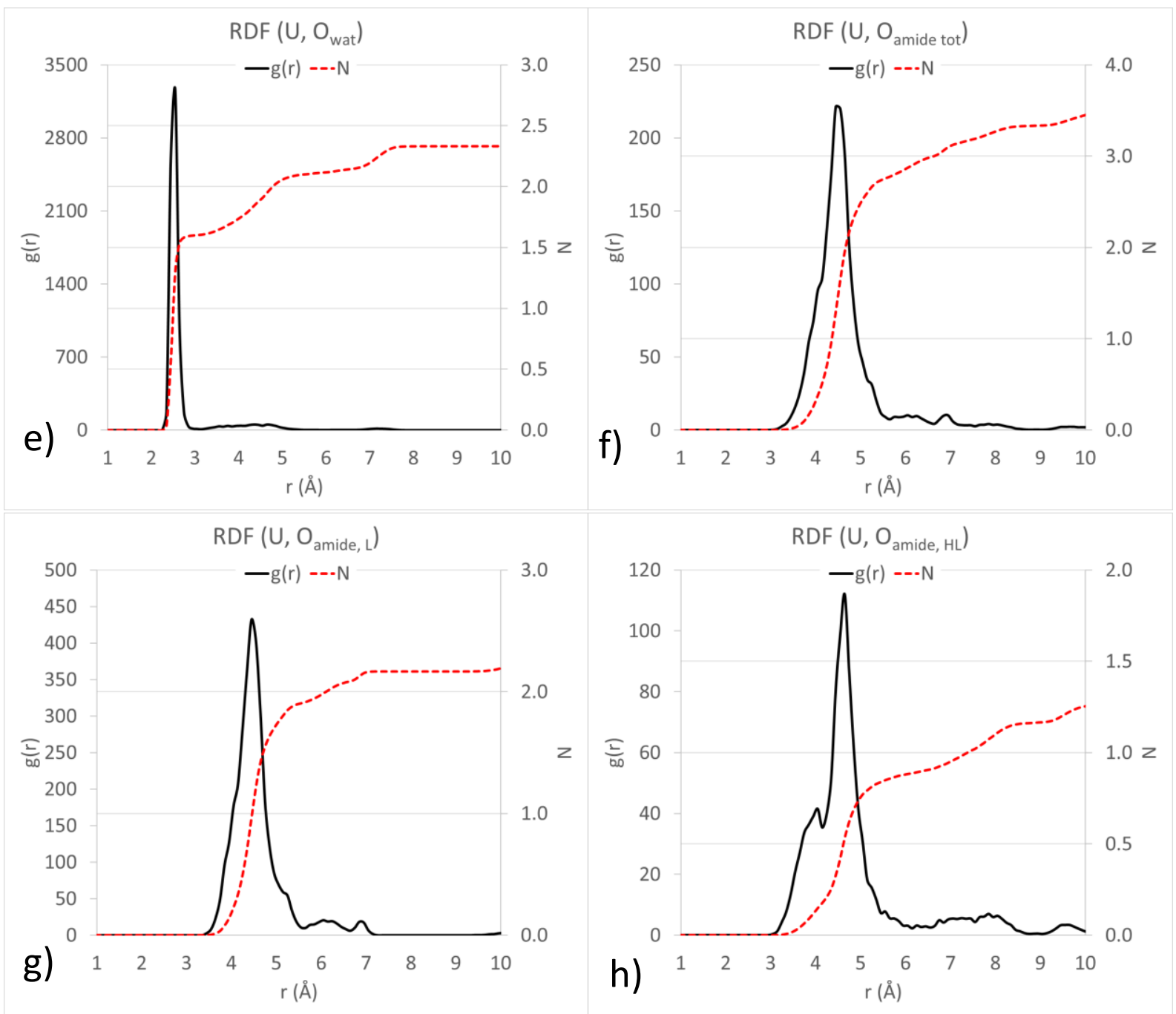

Figure S.3. The Radial Distribution functions (RDFs) centred on uranium atoms with: a) all oxygen atoms; b) deprotonated oxygen atoms of deprotonated extractants (L); c) double bonded oxygen atoms of protonated extractants $(\mathrm{HL})$; d) oxygen atom bearing the acid proton of protonated extractants ( $\mathrm{HL})$; e) oxygen atoms from water molecules; e) all oxygen atoms from amide functions; f) oxygen atoms from amide functions of deprotonated extractants (L); and, g) oxygen atoms from amide functions of protonated extractants (HL); 
RDFs centred on amide oxygen atoms

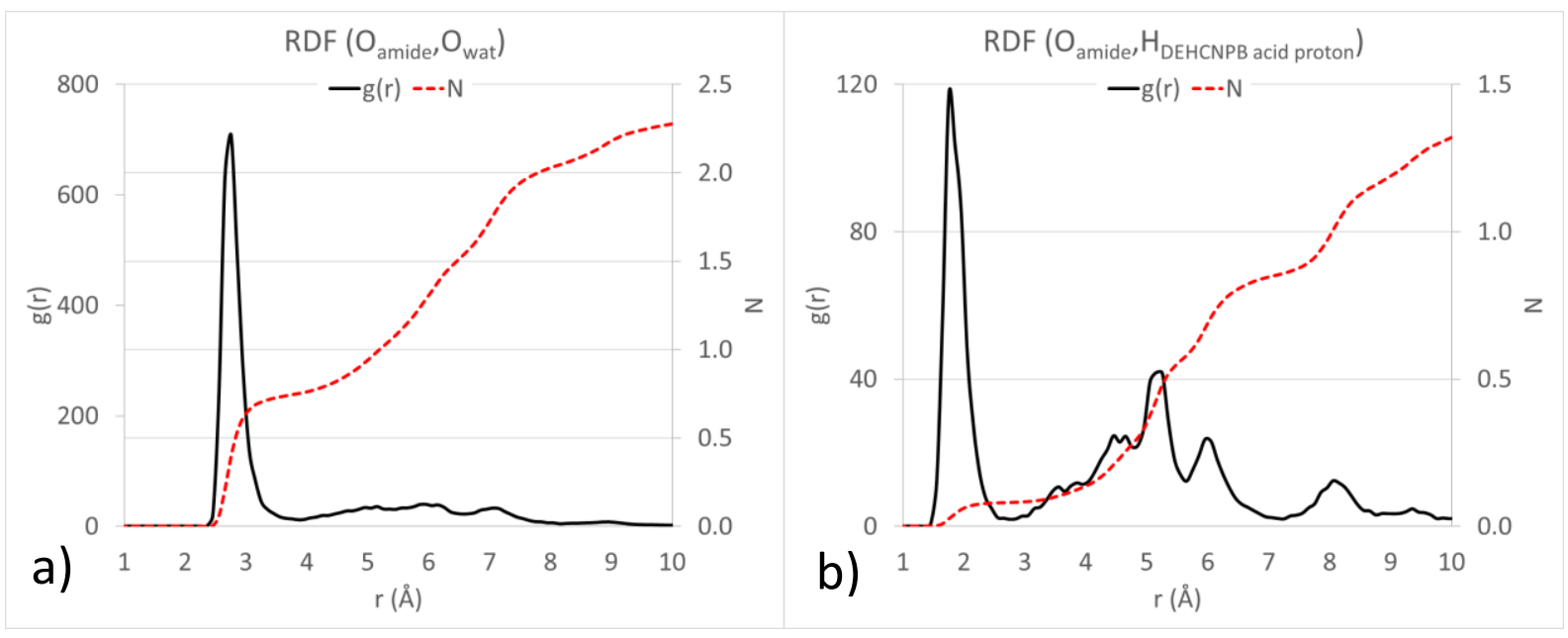

Figure S.4. The Radial Distribution functions (RDFs) centred on amide oxygen atoms in the aggregates containing uranium with: a) oxygen atoms from water molecules; and, b) acid protons of protonated extractants $(\mathrm{HL})$. 


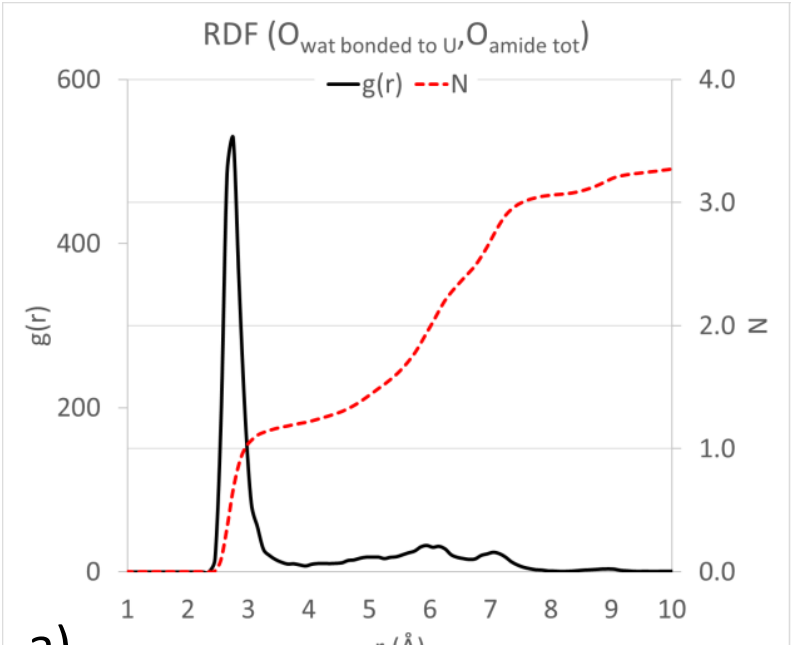

a)
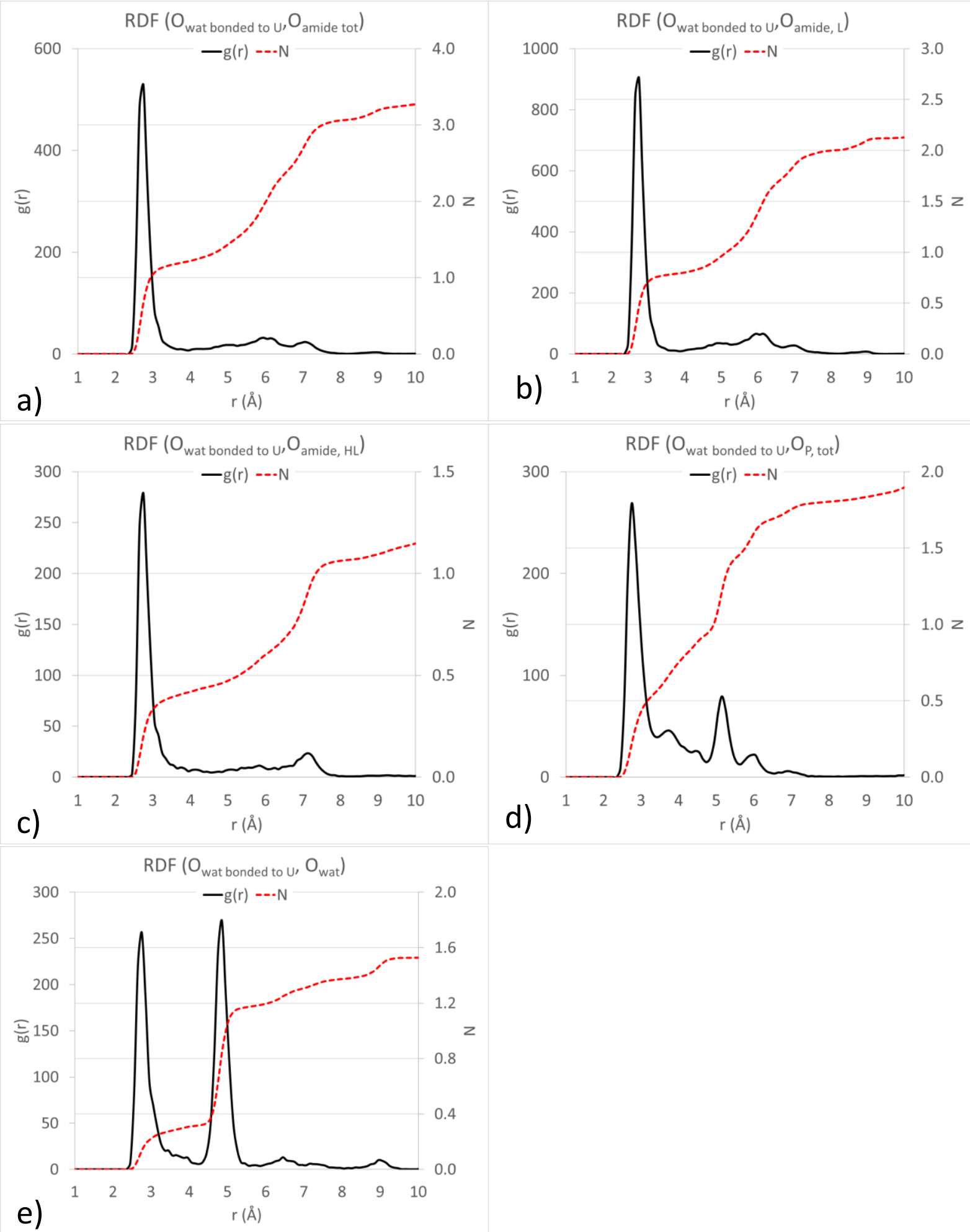

Figure S.5. The Radial Distribution functions (RDFs) centred on oxygen atoms water molecules bonded to uranyl with: a) all oxygen atoms from amide functions; b) oxygen atoms from amide functions of deprotonated extractants (L); c) oxygen atoms from amide functions of protonated extractants ( $\mathrm{HL})$; d) Deprotonated or double bonded oxygen atoms from phosphonate functions; and, e) oxygen atoms from water molecules. 


\section{Equilibrium characterization}

The characterization of the equilibrium, in terms of aggregation, was made by an analysis of the hydrogen bonds formation. First at all, it is relevant to underline that the density is quickly stabilized, in all MD simulations, during the first two nanoseconds. In addition, the temperature is stable during all the dynamic process. Now, as mentioned in the mean paper, the diffusion of DEHCNPB molecules in the n-dodecane organic phase is slow. The formation of aggregates occurs when the polar regions of two or more molecules interact. These interactions, which are hydrogens bonds interactions, are stabilizing in terms of potential energy. The decreasing potential energy during the simulations can be noticed in figure $\mathrm{S} .3$ for the DEHCNPB_08. The decrease of the potential energy is tricky to observe in the most diluted simulations. In this way, it was practical for our study to characterize the formations of aggregates by studying the evolution of hydrogen bonds during the simulation.

To do so, the number of $\mathrm{H}$-bonds were calculated from the integration of RDF functions for six pairs of atoms, this analysis is shown in figure S.7. The distance criteria to get the number of $\mathrm{H}$-bonds correspond to the end of the interaction peak for every RDF. The distances for every pair of atoms are depicted on table S.2.

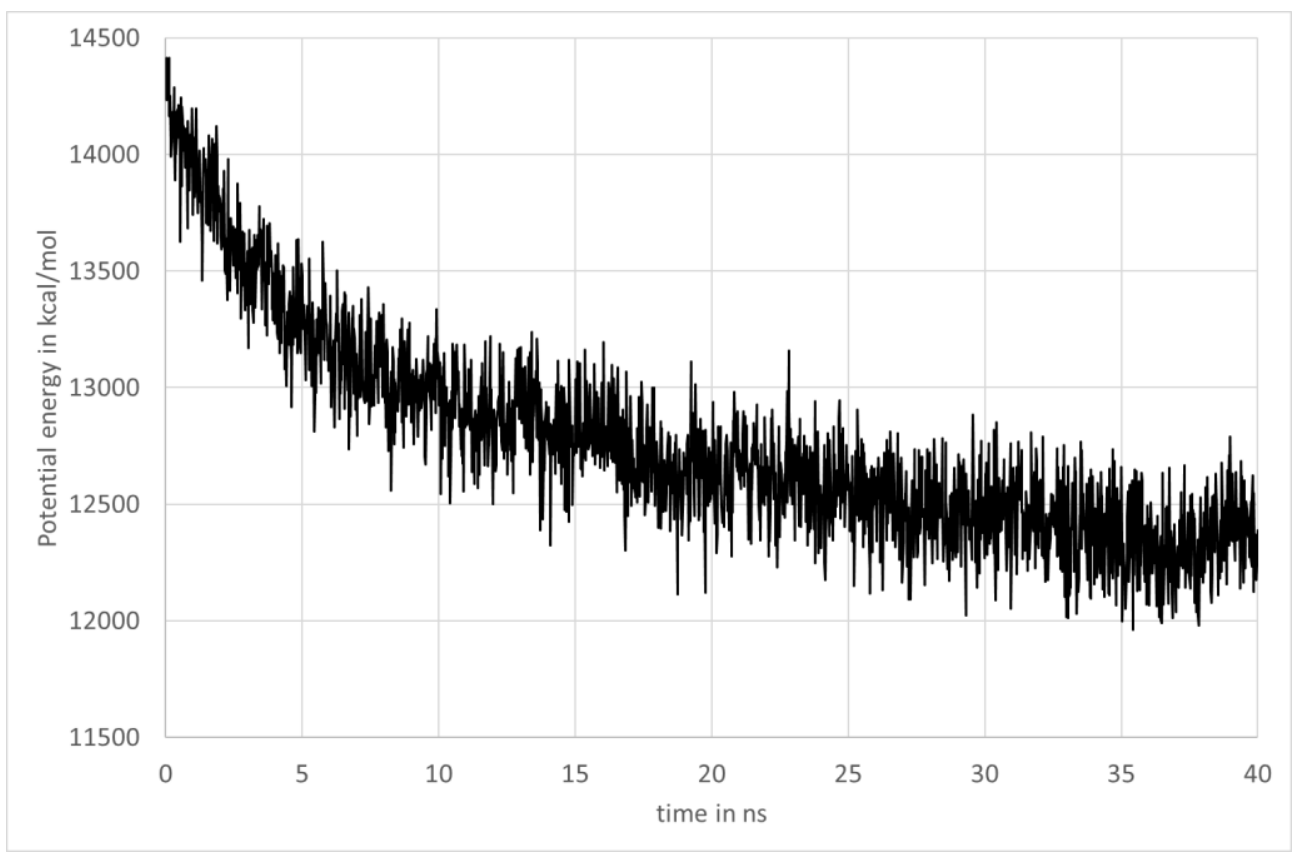

Figure S.6. Evolution of the potential energy for the DEHCNPB_08 simulation 

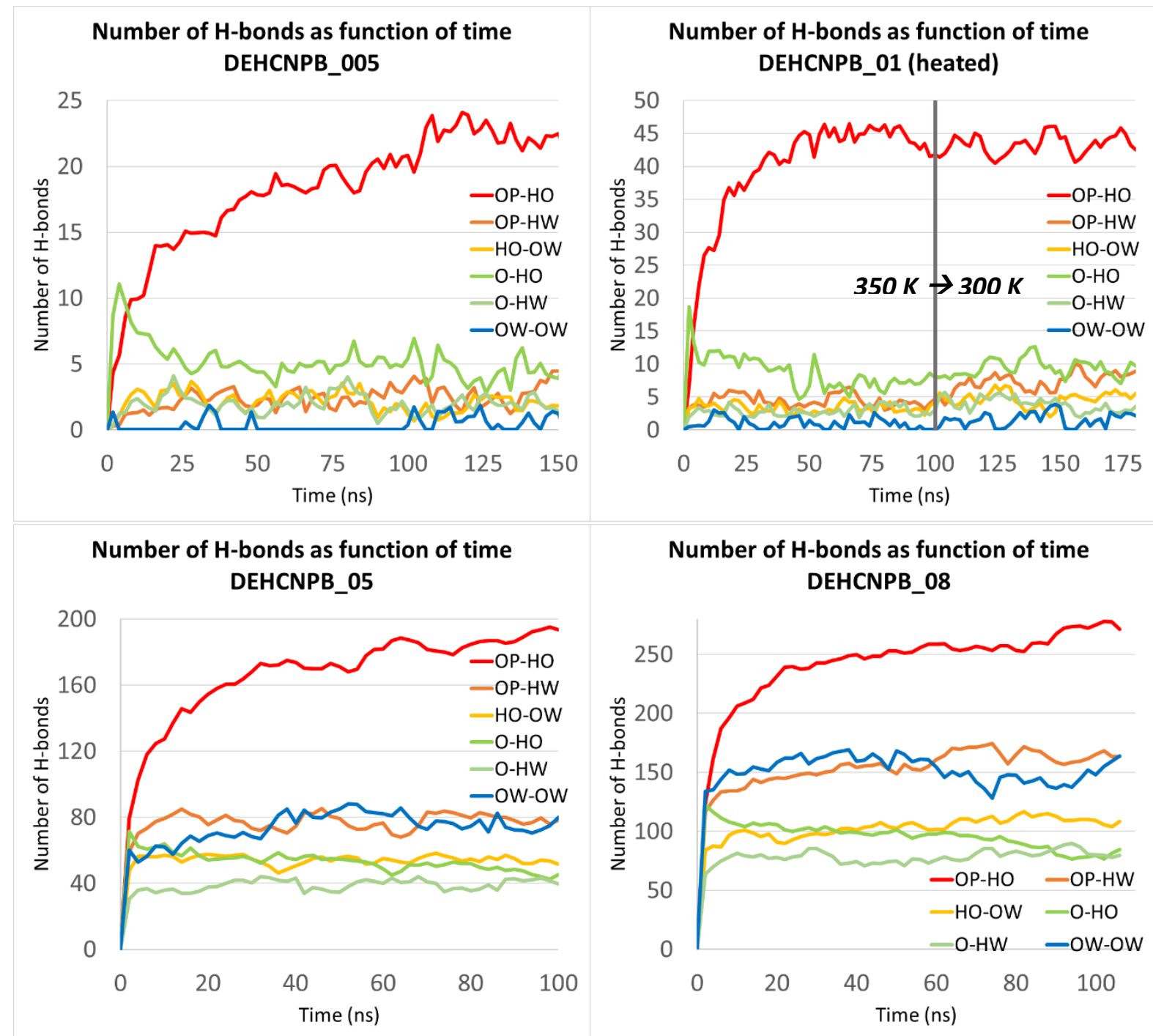

Number of $\mathrm{H}$-bonds as function of time DEHCNPB_01 (not heated)

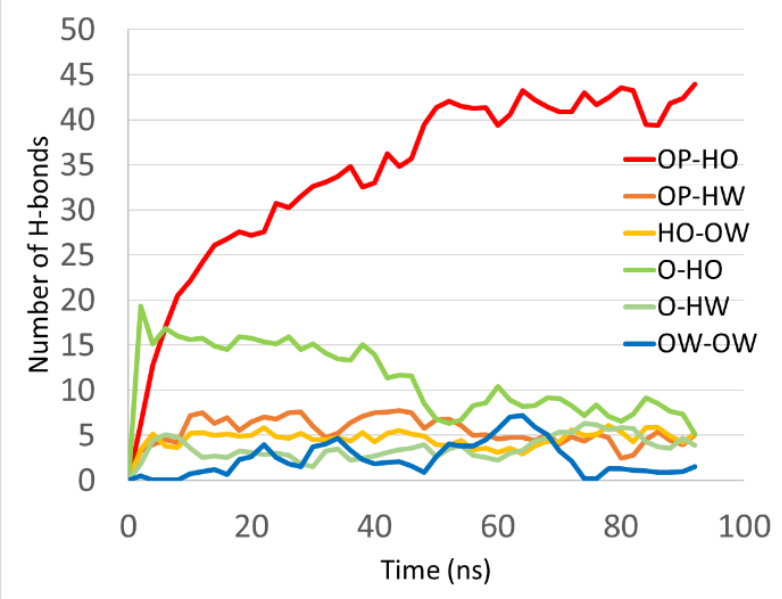

Figure S.7. Evolution of each type of hydrogen bonds in each simulations obtained from RDFs analysis. OP: doubled bonded oxygen atoms of phosphonate; HO: acid protons of phosphonate; $\mathbf{O}$ : amide oxygen atoms; HW: water hydrogen atoms; and $\mathbf{O W}$ : water oxygen atoms. 


\section{Pair of atoms Distance criterion (Å)}

\begin{tabular}{cc}
\hline OP-HO & 2.33 \\
\hline OP-HW & 2.48 \\
\hline HO-OW & 2.33 \\
\hline O-HO & 2.53 \\
\hline O-HW & 2.53 \\
\hline OW-OW & 3.28 \\
\hline
\end{tabular}

Table S.2. Distance criteria used to calculate the number of $\mathrm{H}$-bonds in the simulation boxes from RDFs analysis. OP: doubled bonded oxygen atoms of phosphonate; HO: acid protons of phosphonate; O: amide oxygen atoms; HW: water hydrogen atoms; and OW: water oxygen atoms.

\section{DEHCNPB charges}

\section{Protonated DEHCNPB (HL)}

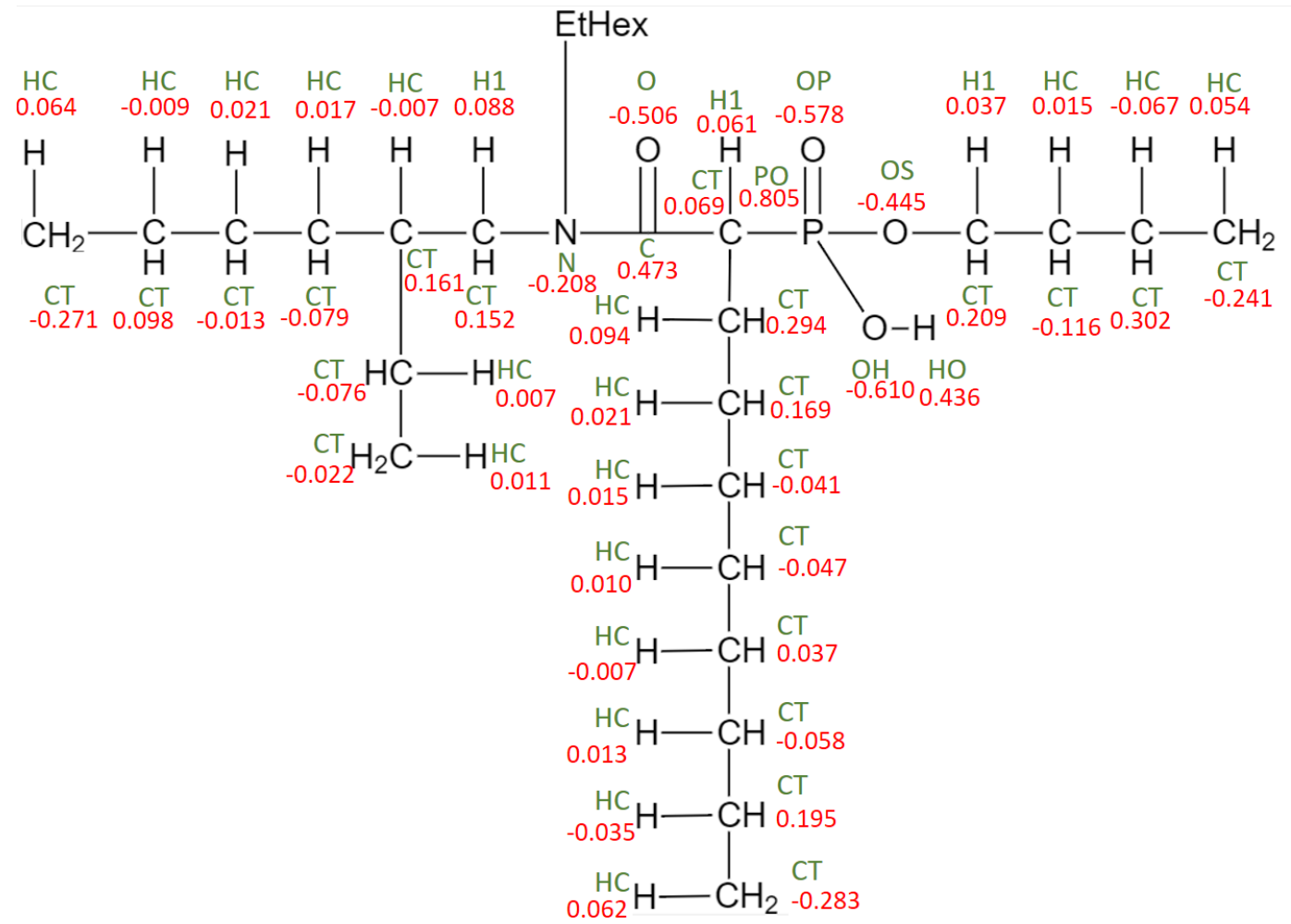




\section{Deprotonated DEHCNPB (L)}

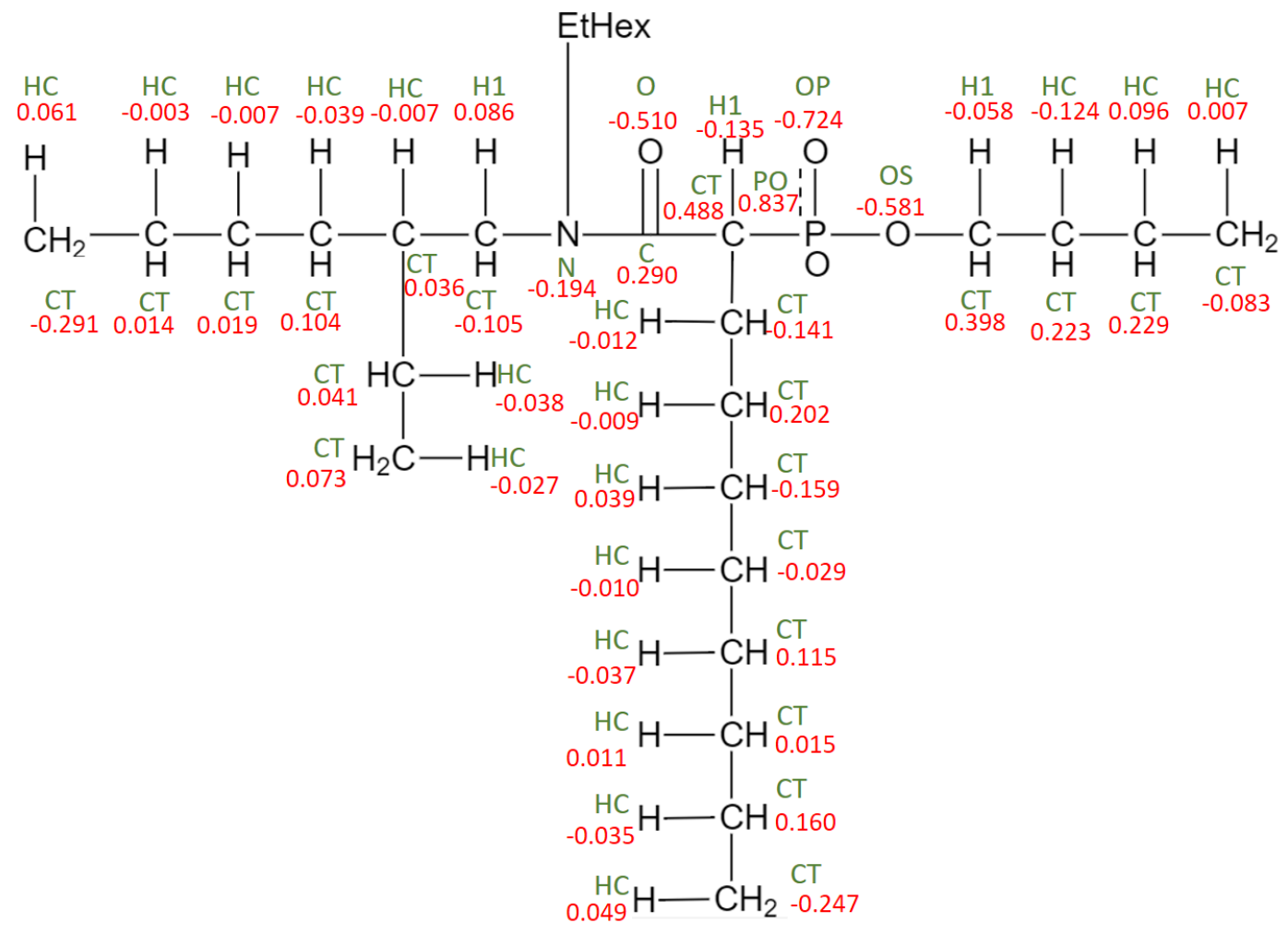

\section{References}

(1) Cornell, W. D.; Cieplak, P.; Bayly, C. I.; Gould, I. R.; Merz, K. M.; Ferguson, D. M.; Spellmeyer, D. C.; Fox, T.; Caldwell, J. W.; Kollman, P. A. A Second Generation Force Field for the Simulation of Proteins, Nucleic Acids, and Organic Molecules. J. Am. Chem. Soc. 1995, 117 (19), 5179-5197. https://doi.org/10.1021/ja00124a002.

(2) Wang, J.; Kollman, P. A. Automatic parameterization of force field by systematic search and genetic algorithms. Journal of Computational Chemistry 2001, 22 (12), 1219-1228. https://doi.org/10.1002/jcc.1079.

(3) Siu, S. W. I.; Pluhackova, K.; Böckmann, R. A. Optimization of the OPLS-AA Force Field for Long Hydrocarbons. J. Chem. Theory Comput. 2012, 8 (4), 1459-1470. https://doi.org/10.1021/ct200908r.

(4) Wang, J.; Wolf, R. M.; Caldwell, J. W.; Kollman, P. A.; Case, D. A. Development and Testing of a General Amber Force Field. Journal of Computational Chemistry 2004, 25 (9), 1157-1174. https://doi.org/10.1002/jcc.20035.

(5) Meagher, K. L.; Redman, L. T.; Carlson, H. A. Development of Polyphosphate Parameters for Use with the AMBER Force Field. Journal of Computational Chemistry 2003, 24 (9), 1016-1025. https://doi.org/10.1002/jcc.10262. 